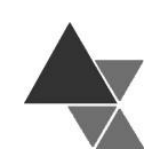

\title{
Oferta de preparações culinárias e alimentos regionais e da sociobiodiversidade na alimentação escolar: um estudo na Região Sul do Brasil
}

\author{
Mariana Werlang Girardi' ${ }^{1}$ Rafaela Karen Fabri², Vitória Uliana Bianchini ${ }^{3}$, Suellen \\ Secchi Martinelli ${ }^{4}$ e Suzi Barletto Cavalli ${ }^{5}$
}

O presente estudo teve como objetivos identificar e analisar a utilização de alimentos e preparações culinárias regionais e alimentos da sociobiodiversidade na alimentação escolar de municípios representantes das mesorregiões da região Sul do Brasil, de acordo com a modalidade de aquisição (licitação ou chamada pública). Trata-se de estudo transversal, exploratório e com abordagem quanti-qualitativa conduzido em quatro etapas. A primeira etapa definiu uma amostra de 21 municípios, representantes de todas as mesorregiões da Região Sul do Brasil. A etapa de coleta de dados, realizada no ano de 2015, consistiu na condução de entrevistas semiestruturadas com 162 atores do PNAE para identificação dos alimentos e preparações reconhecidos como regionais; e no levantamento de cardápios e processos de aquisição de alimentos para posterior identificação da presença de alimentos regionais e da sociobiodiversidade. A etapa de tratamento dos dados consistiu na elaboração das listas de alimentos regionais (entrevistas), e na organização de outras duas listas, de alimentos regionais e da sociobiodiversidade, a partir de documentos oficiais, totalizando três listas para comparação. Para a etapa de análise dos dados, utilizou-se os softwares MaxQDA $®$ e Microsoft Excel ${ }^{\circledR}$ para identificar e analisar com medidas de estatística descritiva a presença dos alimentos nos documentos analisados, bem como a via de fornecimento por qual esses seriam adquiridos (mercado convencional ou agricultura familiar). Quanto aos resultados, dos 142 itens analisados, 76 (53,5\%) estavam presentes nos processos de aquisição de alimentos e nos cardápios da alimentação escolar, dos quais 47,7\% estavam presentes em processos de chamada pública. Constatou-se elevada variação da diversidade de itens entre os municípios, tanto para a análise da variedade de alimentos e preparações culinárias declarados como regionais $(M d=15 ; \mathrm{DP}=7,2)$, quanto para a análise de variação das preparações culinárias e alimentos regionais e da sociobiodiversidade nos processos de aquisição $(M d=18$; $\mathrm{DP}=5,9)$. Dos 123 produtos passíveis de serem identificados em processos de aquisição, 12 equivaleram a aproximadamente $60 \%$ do total de presenças. Dos 35 produtos da sociobiodiversidade, apenas $3(8,5 \%)$ estavam presentes nos processos de aquisição ou nos cardápios. Considerando que o uso de alimentos variados e que respeitem a cultura e as tradições é preconizado pelo PNAE, sugere-se intensificação de iniciativas visando a ampliação da diversidade dos alimentos e preparações regionais e produtos da sociobiodiversidade na alimentação escolar na Região Sul. Sugere-se, por fim, intensificar o incentivo

${ }^{1}$ Mestra em Nutrição pelo Programa de Pós-Graduação em Nutrição da Universidade Federal de Santa Catarina (PPGN-UFSC). Endereço para correspondência: Universidade Federal de Santa Catarina, Centro de Ciências da Saúde, Departamento de Nutrição, Programa de Pós-Graduação em Nutrição. Campus Reitor João David Ferreira Lima, Trindade. CEP: 88040-900. Florianópolis, SC, Brasil. Telefone: 48 3721.2229.E-mail: marianawgirardi@gmail.com

${ }^{2}$ Doutoranda em Nutrição pelo Programa de Pós-Graduação em Nutrição da Universidade Federal de Santa Catarina (PPGN-UFSC) e membro do Núcleo de Pesquisa de Nutrição em Produção de Refeições (NUPPRE).

3 Mestra em Nutrição pelo Programa de Pós-Graduação em Nutrição da Universidade Federal de Santa Catarina (PPGN-UFSC).

${ }^{4}$ Professora Adjunta do Departamento de Nutrição da Universidade Federal de Santa Catarina (PPGN-UFSC) e membro do Núcleo de Pesquisa de Nutrição em Produção de Refeições (NUPPRE).

${ }^{5}$ Professora Associada do Departamento de Nutrição e do Programa de Pós-Graduação em Nutrição da Universidade Federal de Santa Catarina (UFSC) e membro do Núcleo de Pesquisa de Nutrição em Produção de Refeições (NUPPRE). 
à aquisição de alimentos da agricultura familiar via chamada pública e promover a capacitação de atores do PNAE para a aquisição desses alimentos.

Palavras-chave: alimentação escolar, programas e políticas de nutrição e alimentação, políticas públicas, hábitos alimentares.

\section{Regional Foods and Preparations and Sociobiodiversity Food Products in School Feeding: a Study in the South Region of Brazil}

The aim of this study was to identify and analyse the use of regional foods and regional preparations as well as the use of sociobiodiversity food products in school feeding programs in municipalities representing the mesoregions of the South of Brazil, considering the form of acquisition (conventional market or from family farming). This is a transversal and exploratory study, using a quantitative and qualitative approach and was conducted in four phases. A sample of 21 municipalities was defined in the first phase. The municipalities represented all the mesoregions of the South Region of Brazil. The phase of data collection was conducted in 2015 when 162 actors of the National School Feeding Program (PNAE) were consulted through semi-structured interviews in order to identify the foods and preparations recognized as "regional". In addition, menus and processes of food acquisition were collected within the municipalities, with the intention to subsequently verify the presence of regional and sociobiodiversity food products. The phase of data treatment consisted on elaboration of three lists for comparison purposes: one of the regional foods with information received during the interviews and two others collected from official documents and listing regional and sociobiodiversity food products. The last phase was data analysis. MaxQDA® and Microsoft Excel ${ }^{\circledR}$ softwares were used to identify and analyse through descriptive statistic measures the presence of regional foods in the analysed documents, as well as where the food was supplied from (conventional market or family farming). Of the 142 analysed items, 76 (53.5\%) were included in processes of food acquisition and in the school feeding menus. Out of these, $47.7 \%$ were included in acquisition processes from family farming. The results show great variation of item diversity among the municipalities, either in the analysis of the variety of foods and preparations recognized as "regional" (median=15; $\mathrm{SD}=7.2$ ), and in the analysis of the variation of the regional foods and preparations and sociobiodiversity food products in the acquisition processes (median=18; $\mathrm{SD}=5.9$ ). The 12 items that appear more often in the acquisition processes were responsible for about $60 \%$ of the total appearances. Out of the 35 sociobiodiversity food products, only $3(8.5 \%)$ were included in the acquisition processes or in the menus. Considering that the National School Feeding Program (PNAE) recommends the use of a diversity of food items and items that respect regional food culture, the study suggests an intensification of initiatives aiming at increasing the diversity of regional foods and preparations, as well as sociobiodiversity food products in school feeding in the South Region of Brazil. The study also suggests increasing the incentive of food acquisition from family farming, as well as promoting the PNAE actors capability of acquiring these products.

Keywords: school feeding, nutrition programs and policies, public policies, food habits.

\section{INTRODUÇÃO}

A dependência direta e indireta e os impactos da nutrição humana sobre a biodiversidade têm sido cada vez mais conhecidos e documentados pelas áreas da saúde $[1,2]$, da agricultura ${ }^{[3]}$ e do meio-ambiente $[4,5]$. A forma como os alimentos têm sido produzidos e consumidos afeta os ecossistemas e a dieta das populações, levando à sua simplificação e à dependência de um número limitado de alimentos, bem como à desvalorização dos alimentos produzidos localmente ${ }^{[{ }]}$. Desse modo, nutrição, perda da biodiversidade e garantia da segurança alimentar e nutricional (SAN) são temas indissociáveis ${ }^{[7]}$, de forma que a abordagem conjunta é essencial no âmbito das políticas públicas[4]. 
O Brasil detém de 15 a 20\% da biodiversidade mundial[8,9], atualmente ameaçada pelo modelo de produção agrária do país, voltado ao agronegócio empresarial[10,11]. Esse tipo de produção envolve processos altamente mecanizados, monocultura em larga escala e uso intensivo de insumos industriais como agrotóxicos e sementes transgênicas $\left.{ }^{[}\right]$, além de ampla dependência de combustíveis fósseis ${ }^{[12]}$. Desse modo, consiste em um sistema de produção insustentável[13]. Além disso, os alimentos produzidos por esse modelo estão intimamente ligados ao atual quadro de saúde e nutrição da população brasileira, caracterizado por elevados índices de sobrepeso e de doenças crônicas não transmissíveis associadas a uma considerável prevalência de déficits nutricionais ${ }^{[1,15]}$.

Recente publicação da Organização das Nações Unidas para Agricultura e Alimentação ${ }^{[16]}$ associa a diminuição do consumo de preparações culinárias tradicionais baseadas em alimentos frescos, preparados e consumidos localmente à ocorrência de transição nutricional em toda a América Latina, enquanto a valorização da agricultura local teria o potencial de aumentar a biodiversidade ${ }^{[17]}$ e promover uma alimentação mais saudável à população.

No Brasil, a Política Nacional de Segurança Alimentar e Nutricional (PNSAN) apresenta como uma de suas diretrizes "promover sistemas sustentáveis (...) de produção e distribuição de alimentos que respeitem a biodiversidade e fortalecam a agricultura familiar' ${ }^{2}[18]$. Desse modo, é necessário restabelecer os vínculos da produção e do consumo de alimentos com seu entorno socioambiental[7,19]. Nessa perspectiva, um sistema alimentar que favorece esse tipo de abordagem é considerado sensível à nutrição ${ }^{[20]}$.

No Brasil, o Programa Nacional de Alimentação Escolar (PNAE) é considerado pelo United Nations System Standing Committee on Nutrition (UNSCN) a iniciativa que mais se destaca dentre as políticas brasileiras em relação ao critério de sensibilidade à nutrição, obtendo a melhor avaliação dentre as políticas comparadas ${ }^{[2]}$. O PNAE tem sido um dos responsáveis pelo reconhecimento internacional do sucesso brasileiro no combate à má nutrição e à pobreza de forma integrada e sustentável[22], em consonância com princípios da PNSAN. Essas experiências têm sido compartilhadas com dezenas de países com os quais o Brasil faz cooperação Sul-Sul, sobretudo países latino-americanos e africanos, principalmente por meio do Centro de Excelência contra a Fome do Programa Mundial de Alimentos das Nações Unidas ${ }^{[23]}$.

A alimentação escolar no Brasil gera um mercado institucional de alimentos importante para o escoamento da produção agrícola familiar ${ }^{[24]}$, frente à obrigatoriedade de compra da agricultura familiar, via chamada pública, estabelecida pela legislação ${ }^{6[25]}$. Dessa forma, permite-se o avanço na flexibilização das legislações em prol da dinamização da economia local e regional[26].

Ressalta-se que políticas que apoiem a proteção e promoção da biodiversidade e que possuam abordagens sensíveis à nutrição, como PNAE, são fundamentais para o alcance dos Objetivos do Desenvolvimento Sustentável (ODS) ${ }^{[21]}$. A produção da agricultura familiar é a que melhor proporciona a conservação, o uso sustentável e o manejo da biodiversidade [27], sendo compatível com a promoção de hábitos alimentares saudáveis por meio do estímulo ao consumo de alimentos frescos e da utilização de alimentos regionais que promovam a sociobiodiversidade ${ }^{[28]}$.

A inclusão de alimentos regionais e da sociobiodiversidade no cardápio das escolas contribui para o aumento da produção agrícola familiar; valorização dos produtos locais; estímulo à economia local; ampliação da qualidade da alimentação escolar; promoção da biodiversidade local; conservação de recursos naturais ${ }^{[29]}$ e promoção de justiça social e desenvolvimento local sustentável[28], devendo por isso ser incentivada de diversas formas.

No entanto, alguns estudos em municípios brasileiros identificaram baixa oferta de alimentos regionais na alimentação escolar [30,31]. O estímulo ao consumo de alimentos e preparações culinárias regionais no âmbito da alimentação escolar é uma tarefa complexa devido à falta de consenso sobre quais alimentos e preparações representam a cultura alimentar e os hábitos alimentares locais ${ }^{[32]}$. Além disso, a lista de alimentos regionais apresentada no documento oficial existente ${ }^{[3]}$ é demasiadamente

municípios e estados devem ser utilizados na compra de alimentos da agricultura familiar[25].
6 No mínimo 30\% dos recursos repassados pelo Fundo Nacional de Desenvolvimento da Educação/Ministério da Educação (FNDE/MEC) aos 
abrangente e inespecífica, podendo não retratar a realidade local[32].

Por fim, na literatura científica há escassez de estudos que avaliem a inclusão de alimentos e preparações culinárias regionais na alimentação escolar e não foi identificado nenhum estudo que conduzisse análises do uso de alimentos da sociobiodiversidade. Nesse contexto, o presente estudo teve como objetivos identificar e analisar o uso de alimentos e preparações culinárias regionais e alimentos da sociobiodiversidade na alimentação escolar de municípios da região Sul do Brasil.

\section{METODOLOGIA}

Trata-se de um estudo transversal, exploratório e com abordagem quanti-qualitativa realizado em municípios representativos das mesorregiões da Região Sul do Brasil. A metodologia do presente estudo foi dividida nas quatro etapas a seguir: definição da amostra, coleta de dados, tratamento e análise dos dados.

$\mathrm{Na}$ etapa de definição da amostra, buscou-se uma amostra representativa de todas as mesorregiões ${ }^{7}$ dos três estados da Região Sul, quais sejam: Paraná, Santa Catarina e Rio Grande do Sul. Esses estados são subdivididos em dez, seis e sete mesorregiões, respectivamente, totalizando 23 municípios. Os critérios para a seleção dos municípios por mesorregião foram intencionais e com base na experiência prévia das autoras em pesquisas sobre aquisição de alimentos para a alimentação escolar e alimentos regionais ${ }^{[2,32,35]}$.

Desse modo, definiu-se dois critérios para exclusão de municípios da amostra, quais sejam: possuir menos de 20 mil habitantes - dada a estrutura simplificada dos serviços que dão suporte ao PNAE, incluindo ausência de nutricionista - e possuir mais de 50 mil habitantes - dada a possível estrutura e logística mais complexas na execução do PNAE e por não haver nessa faixa populacional municípios representantes para todas as mesorregiões. Um critério complementar para inclusão de municípios na amostra foi possuir, em seu quadro funcional, nutricionista designado como responsável técnico.

Do grupo de municípios que atenderam aos critérios de inclusão e exclusão, selecionou-se um representante por mesorregião por meio de amostragem aleatória simples. Em caso de recusa do município em participar da pesquisa, uma nova seleção nos mesmos moldes foi realizada. Houve recusa de participação em todos os municípios selecionados para duas mesorregiões do estado do Paraná, sendo elas: Centro Sul e Norte Pioneiro. Desse modo, a amostra final deste estudo ficou definida por 21 municípios da Região Sul do Brasil (Figura 1).

Figura 1. Região Sul do Brasil em mesorregiões geográficas pesquisadas, 2015.

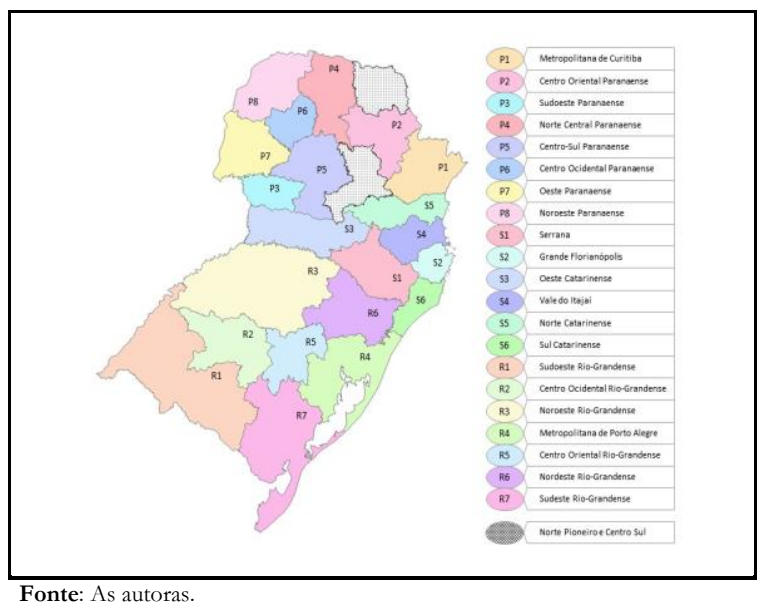

A mesorregião é uma subdivisão dos estados brasileiros que congrega diversos municípios de uma área geográfica com similaridades econômicas e sociais ${ }^{[34]}$. 
A etapa de coleta de dados ocorreu entre os meses de março e novembro do ano de 2015 e consistiu-se em entrevistas semiestruturadas e no levantamento de documentos oficiais (cardápios e processos de aquisição de alimentos) do ano de 2014. Esse levantamento foi realizado in loco nas prefeituras e por busca em seus respectivos portais na internet.

Foram coletados 88 processos de aquisição, dos quais 43 tratavam-se de aquisições por meio de chamadas públicas e 45 de aquisições por meio de licitações, em sua maioria realizados na modalidade pregão. Além disso, foram coletados todos os cardápios das escolas do ensino fundamental do ano de 2014. Ressalta-se que a configuração dos cardápios em cada município é variável, podendo existir cardápios mensais, bimestrais, semestrais, dentre outras possibilidades. Para aqueles municípios que não disponibilizaram algum documento do ano de 2014, utilizou-se para análise documentos do ano de 2015 $(n=2)$.

As entrevistas foram realizadas com atores sociais do PNAE dos 21 municípios da amostra, tendo como objetivo obter informações acerca do que estes consideravam alimentos e preparações culinárias regionais. Foram entrevistados agentes relacionados ao setor da alimentação escolar (nutricionistas, cozinheiros, conselheiros de alimentação escolar) e ao setor da agricultura familiar (agricultores, representantes de cooperativas e de assistência técnica). Outros possíveis entrevistados de cada setor foram selecionados por meio da técnica bola de neve, na qual os entrevistados denominam outros possíveis entrevistados de acordo com a temática de interesse ${ }^{[3]}$. Desse modo, o número de entrevistados por município variou entre sete e nove agentes, totalizando 162.

Para fins deste estudo, considerou-se alimentos e preparações culinárias regionais aqueles com peculiaridades históricas, culturais e socioeconômicas relacionadas ao local de origem da produção, técnicas de produção, uso de recursos naturais e tecnológicos, crença, religião, educação e etnia que influenciam diretamente a sua produção e o seu consumo. Por alimentos da sociobiodiversidade, considerou-se que são aqueles gerados a partir de recursos da biodiversidade nativa inter-relacionados com sistemas socioculturais, sendo voltados à formação de cadeias produtivas de interesse de povos e comunidades tradicionais e de agricultores familiares.

As entrevistas foram conduzidas por pesquisadores treinados com auxilio de tablets para gravação de áudio e para o registro escrito das respostas (software Epicollect+ Beta ${ }^{\circledR}$ ). Posteriormente as entrevistas foram transcritas em verbatim e organizadas por estado, município, cargo e código do agente entrevistado.

A etapa de tratamento dos dados compreendeu a elaboração de um grupo de listas de alimentos regionais por município e a organização de listas de alimentos regionais e da sociobiodiversidade baseadas em documentos oficiais, conforme Quadro 1.

Quadro 1. Descrição das listas utilizadas para a análise

\begin{tabular}{|l|c|l|c|}
\hline \multicolumn{1}{|c|}{ Denominação } & $\begin{array}{c}\text { Segmentação } \\
\text { geográfica }\end{array}$ & \multicolumn{1}{|c|}{ Fonte } & Ano \\
\hline Listas de Regionais - Autoras & Municipal & Entrevistas com atores do PNAE & 2015 \\
\hline Lista de Regionais - Oficial (MS) & Regional & Ministério da Saúde (MS) (BRASIL, 2015) & 2015 \\
\hline Lista da Sociobiodiversidade & Estadual & $\begin{array}{l}\text { Ministérios do Meio Ambiente (MMA) e do Desenvolvimento } \\
\text { Social e Combate à Fome (MDS) (BRASIL, 2016a) }\end{array}$ & 2016 \\
\hline
\end{tabular}

Para a realização dessa etapa, inicialmente procedeu-se a análise das entrevistas, tendo como objetivo identificar quais alimentos e preparações culinárias constavam como citados mais de uma vez como regionais pelos atores entrevistados em âmbito municipal. Esses alimentos e preparações culinárias, uma vez identificados, passaram a compor listas de alimentos e preparações regionais por município, dando origem, portanto, a 21 listas. Para fins de descrição da 
metodologia, convencionou-se denominar esse grupo de Listas de Regionais - Autoras.

Para a organização das demais listas, extraiu-se da publicação oficial do Ministério da Saúde (MS), denominada Alimentos Regionais Brasileiros[33], os alimentos considerados regionais na Região Sul. Dessa forma, obteve-se a Lista de Regionais - Oficial MS.

Por fim, extraiu-se da Portaria Interministerial dos Ministérios do Meio Ambiente (MMA) e do Desenvolvimento Social e Combate à Fome (MDS) ${ }^{8}$ no $163 / 2016$ os alimentos considerados da sociobiodiversidade para cada um dos estados da Região Sul (Paraná, Santa Catarina e Rio Grande do Sul). Dessa forma, obteve-se a Lista da Sociobiodiversidade.

$\mathrm{Na}$ etapa de análise dos dados, utilizou-se as três listas selecionadas para identificar a presença de alimentos e preparações culinárias nos documentos oficiais levantados por município (processos de aquisição e cardápios da alimentação escolar) por meio do softwrare para análise de dados qualitativos MaxQDA® (versão 12.2.1).

No processo de identificação, vocábulos que representavam um único alimento ou preparação foram compostos por palavras sinônimas, por exemplo: peixe, pescada e pescado. Para os casos onde havia a possibilidade de "falso positivo" a identificação do alimento foi feita manualmente. Por exemplo, se o alimento regional era mandioca, o software poderia identificar indevidamente farinha de mandioca quando essa estava presente como ingrediente de algum outro produto (não sendo adquirida nos processos licitatórios).

A partir das Listas de Regionais - Autoras identificou-se quais alimentos estavam presentes nos processos de aquisição e quais preparações culinárias estavam presentes nos cardápios dos 21 municípios pesquisados (excluiu-se bebidas alcoólicas citadas como regionais). Nessa etapa, a comparação foi feita por município. Assim, a lista de alimentos regionais elaborada especificamente para um dos municípios foi utilizada para identificar alimentos e preparações nos

\footnotetext{
${ }^{8}$ Atual Ministério do Desenvolvimento Social e Agrário (MDSA).

9 Arroz, bolacha/biscoito caseiro, bolo, canjica, canjiquinha, chimia/doce de fruta, cuca/cuque, feijão, macarrão/macarronada/massa, pão caseiro, polenta e respectivas preparações.
}

processos de aquisição e nos cardápios desse mesmo município.

As preparações culinárias regionais que poderiam ser adquiridas prontas ou preparadas nas escolas, a exemplo de cuca, do pão caseiro e da massa caseira, foram identificadas nos processos de aquisição e nos cardápios. O mesmo aconteceu para o arroz e o feijão, quando citados como regionais, por serem preparações tradicionais da dieta brasileira ${ }^{[37]}$.

No caso de preparações culinárias regionais cujos ingredientes base não haviam sido citados pelos entrevistados, verificou-se a sua presença nos processos de compra. Por exemplo, se os agentes do município citaram polenta como preparação culinária regional, cujo ingrediente base é o fubá e/ou a farinha de milho, esses foram rastreados nos processos de aquisição assumindo-se que foram utilizados no preparo da polenta.

Devido ao fato de que alguns alimentos podem também ser preparações culinárias, a exemplo da combinação arroz e feijão mencionada acima, classificou-se o grupo de Listas de Regionais - Autoras em três subgrupos para permitir a análise dos dados, sendo eles: alimentos $(\mathrm{n}=112)$; alimentos ou preparações $(\mathrm{n}=11)^{9}$; e preparações $(\mathrm{n}=19)^{10}$.

A análise da presença dos alimentos constantes na Lista da Sociobiodiversidade foi realizada a partir dos processos de aquisição, por estados (não por município), conforme divisão da Portaria Interministerial no 163/2016[38]. A seguir, buscou-se identificar os alimentos da Lista de Regionais - Oficial MS nos processos de compra de todos os municípios. Neste caso, a lista foi utilizada por região (Região Sul), pois a publicação Alimentos Regionais Brasileiros[33] é segmentada geograficamente pelas cinco regiões brasileiras (Norte, Nordeste, Centro-oeste, Sudeste e Sul).

A presença de alimentos e preparações regionais foi analisada por meio de estatística descritiva com auxílio do software Microsoft Excel® 2013. As principais variáveis de análise foram: as três listas (Grupo A, B e C) e 11 subgrupos; o universo dos itens

\footnotetext{
10 Arroz com galinha, batata-doce caramelizada, broa de milho, canja/sopa de frango, capeletti, carreteiro, chucrute, churrasco, ensopado de mandioca, feijoada, gemuse/guimis, leitão maturado, nhoque de batata, pirão de peixe, porco no tacho, schwarsaur (sopa de sangue), stollen, strudel, virado de feijão.
} 
pesquisados, segmentado em alimentos ou preparações culinárias regionais; as fontes de informação oficiais, segmentada em cardápios e processos de aquisição. Com a finalidade de possibilitar análises sobre a compra da agricultura familiar, os processos de aquisição foram subdivididos em processos de aquisição por licitação ou por chamada pública.

A identificação teve como possíveis resultados as seguintes respostas: (a) alimento presente ou alimento ausente em processo de aquisição; (b) alimento e preparação culinária presente ou alimento e preparação culinária ausente em cardápio. Não foi feita distinção relacionada à quantidade de vezes que determinado alimento ou preparação aparece em documento oficial. Por exemplo, se determinado alimento é identificado em três licitações e outro em uma licitação e duas chamadas públicas, ambos serão identificados apenas como presentes $(n=1)$. Àqueles que não apareceram em documentos oficiais foram atribuídos pesos zero $(n=0)$.

Destaca-se que esta pesquisa faz parte de um projeto maior intitulado "Qualidade da alimentação escolar a partir do fornecimento de alimentos da agricultura familiar: um estudo multicêntrico da Região Sul do Brasil", financiado pelo edital Universal MCTI/CNPq no 14/2012. A pesquisa foi submetida ao Comitê de Ética em Pesquisa, por meio da Plataforma Brasil e aprovada mediante parecer de número 1.002.956.

\section{RESULTADOS}

Foram listados 142 alimentos e preparações para serem identificados nos processos de aquisição de alimentos e nos cardápios da alimentação escolar dos 21 municípios que compuseram a amostra final deste estudo. Esse universo de itens alimentares considera três grupos: Listas de Regionais - Autoras ( $\mathrm{n}=95,66,9 \%$ ); Lista de Regionais - Oficial (MS) ( $\mathrm{n}=30,21,1 \%) ;$ Lista da Sociobiodiversidade ( $\mathrm{n}=35,24,6 \%)$ (Diagrama 1).
Dos 35 alimentos da sociobiodiversidade brasileira da Região Sul do Brasil (Grupo A), todos representam o estado do Paraná, ${ }^{11} 27$ o estado de Santa Catarina $^{12}$ e 25 o estado do Rio Grande do Sul13. O alimento pinhão foi o único comum aos três grupos de alimentos analisados, fazendo parte do subgrupo $\mathrm{A} \cap$ B $\cap$ C. O alimento maracujá foi identificado simultaneamente na Lista da Sociobiodiversidade e no grupo de Listas de Regionais - Autoras, subgrupo A $\cap \mathrm{C}$. A batata-crem foi identificada na Lista da Sociobiodiversidade e na Lista de Regionais - Oficial MS, subgrupo $A \cap B$.

Foram encontrados catorze alimentos comuns à Lista de Regionais - Oficial MS e ao grupo de Listas de Regionais - Autoras, subgrupo $\mathrm{B} \cap \mathrm{C}$, sendo eles: almeirão, banana, batata inglesa, batata-doce, beterraba, maçã, milho, morango, pêssego, radite, repolho, tangerina, tomate e uva. Há, ainda, os três subgrupos dos produtos que fazem parte exclusivamente da Lista da Sociobiodiversidade, da Lista de Regionais - Oficial MS e das Listas de Regionais - Autoras.

Diagrama 1. Universo dos itens analisados e os três grupos que o compõem. Diagrama de Venn

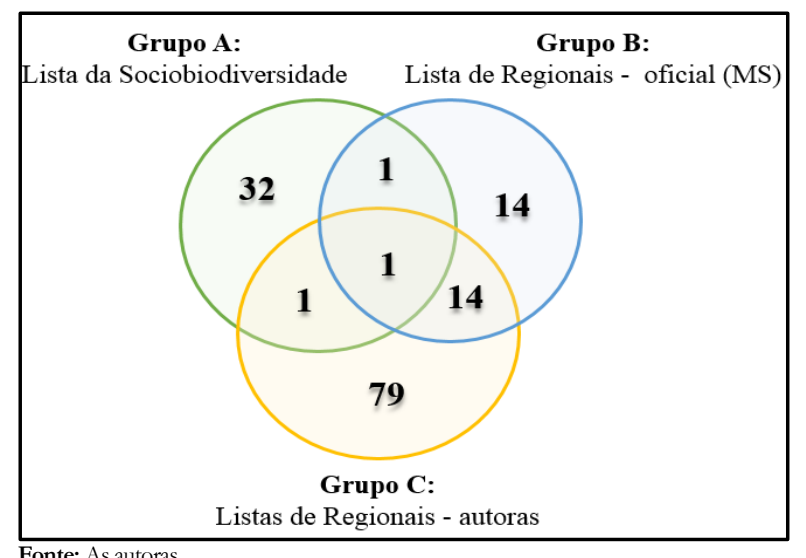

Fonte: As autoras.
$11 \mathrm{O}$ araticum, o caju-do cerrado, a gueroba, o jatobá, a macaúba, a mangaba, o pequi e a taioba são considerados produtos da sociobiodiversidade apenas no estado do Paraná.

12 A guabirova/gabiroba e o jenipapo são considerados produtos da sociobiodiversidade nos estados do Paraná e de Santa Catarina.
${ }^{13}$ Os demais produtos são comuns aos três estados, sendo eles: araçá, aroeirapimenteira, arumbeva, beldroega, butiá, cereja, crem/batata crem, erva-mate, fisalis, goiaba-serrana, jabuticaba, jaracatiá/mamãozinho, jaracatiá/mamão-do-mato, juçara, jurubeba, major-gomes, maracujá, mini-pepininho, murici, ora-pro-nobis, pinhão, pitanga, urucum e uvaia. 
O grupo de Listas de Regionais - Autoras permitiu a identificação os dez alimentos e preparações mais citados pelos municípios como regionais, sendo eles: mandioca $(90,5 \%)$, arroz $(85,7 \%)$, feijão $(81,1 \%)$, alface $(71,4 \%)$, batata-doce $(71,4 \%)$, laranja $(61,9 \%)$, milho $(57,1 \%)$, carne suína $(47,6 \%)$, tangerina $(47,6 \%)$ e carne bovina $(38,1 \%)$.

Em todos os municípios do Paraná e de Santa Catarina, a mandioca foi citada como alimento regional e no Rio Grande do Sul esse alimento foi citado em $71,4 \%$ dos municípios. Os alimentos/preparações arroz e feijão apareceram nos três estados entre os três itens mais citados.

Para maior granularidade das análises, os grupos foram subdivididos em sete subgrupos, conforme Quadro 2.

Quadro 2. Correspondência entre quantidade e percentual de alimentos ou preparações nos grupos e subgrupos (em relação ao universo de itens), a quantidade e o percentual de presença dos alimentos em pelo menos um processo de aquisição e em todos os processos de aquisição

\begin{tabular}{|c|c|c|c|c|c|c|c|}
\hline \multirow{3}{*}{$\begin{array}{l}\text { Grupos e } \\
\text { subgrupos }\end{array}$} & \multirow{3}{*}{ Nome } & \multirow{2}{*}{\multicolumn{2}{|c|}{ Alimentos ou preparações }} & \multicolumn{4}{|c|}{$\begin{array}{l}\text { Presença dos alimentos } \\
\end{array}$} \\
\hline & & & & \multicolumn{2}{|c|}{$\begin{array}{c}\text { Em pelo menos um } \\
\text { processo de aquisição }\end{array}$} & \multicolumn{2}{|c|}{$\begin{array}{c}\text { Em todos os processos de } \\
\text { aquisição }\end{array}$} \\
\hline & & Quantidade & $\%$ & Quantidade & $\%$ & Quantidade & $\%$ \\
\hline $\mathrm{A}$ & Sociobiodiversidade & 35 & 24,65 & 3 & 3,95 & 17 & 4,66 \\
\hline $\mathrm{B}$ & Regionais - oficial (MS) & 30 & 21,13 & 18 & 23,68 & 175 & 47,95 \\
\hline $\mathrm{C}$ & Regionais - autoras & 95 & 66,90 & 62 & 81,58 & 323 & 88,49 \\
\hline $\mathrm{A} \backslash(\mathrm{B} U \mathrm{C})$ & $\begin{array}{c}\text { Sociobiodiversidade, } \\
\text { exclusivamente }\end{array}$ & 32 & 22,54 & 10 & 13,16 & 10 & 2,74 \\
\hline $\mathrm{B} \backslash(\mathrm{A} U \mathrm{C})$ & $\begin{array}{l}\text { Regionais - oficial } \\
\text { (MS), exclusivamente }\end{array}$ & 14 & 9,86 & 4 & 5,26 & 32 & 8,77 \\
\hline$C \backslash(\mathrm{A} U \mathrm{~B})$ & $\begin{array}{c}\text { Regionais - autoras, } \\
\text { exclusivamente }\end{array}$ & 79 & 55,63 & 47 & 61,84 & 174 & 47,67 \\
\hline$A \cap B$ & $\begin{array}{l}\text { Sociobiodiversidade e } \\
\text { Regionais - oficial } \\
\text { (MS), simultaneamente }\end{array}$ & 1 & 0,70 & 0 & 0,00 & 0 & 0,00 \\
\hline$A \cap C$ & $\begin{array}{l}\text { Sociobiodiversidade e } \\
\text { Regionais - autoras, } \\
\text { simultaneamente }\end{array}$ & 1 & 0,70 & 1 & 1,32 & 6 & 1,64 \\
\hline$B \cap C$ & $\begin{array}{l}\text { Regionais - oficial (MS) } \\
\text { e Regionais - autoras, } \\
\text { simultaneamente }\end{array}$ & 14 & 9,86 & 13 & 17,11 & 142 & 38,90 \\
\hline$A \cap B \cap C$ & $\begin{array}{c}\text { Sociobiod. Regionais - } \\
\text { oficial (MS) e Regionais } \\
\text { - autoras, } \\
\text { simultaneamente }\end{array}$ & 1 & 0,70 & 1 & 1,32 & 1 & 0,27 \\
\hline A U B U C & $\begin{array}{c}\text { Totalidade (ou } \\
\text { universo) dos } \\
\text { produtos }\end{array}$ & 142 & 100,00 & 76 & 100,00 & 365 & 100,00 \\
\hline
\end{tabular}

Fonte: As autoras.

Os 35 itens pertencentes ao Grupo A (Lista da Sociobiodiversidade) representam $24,6 \%$ do universo analisado de 142 itens, entretanto, teve apenas $3(9,1 \%$ de 76) desses itens presentes em pelo menos um processo de aquisição. Esses 3 itens estiveram presentes em $17(4,6 \%)$ de todos os processos de aquisição
( $\mathrm{n}=365)$. Os 30 itens pertencentes ao Grupo B (Lista de Regionais - Oficial MS) representam 21,1\% do universo analisado de 142 itens, estando 18 (23,6\% de 76) desses itens presentes em pelo menos um processo de aquisição. Esses 18 itens estiveram presentes em 175 $(47,9 \%)$ de todos os processos de aquisição $(\mathrm{n}=365)$. 
Os 95 itens pertencentes ao Grupo C (Listas de Regionais - Autoras) representam 66,9\% do universo analisado de 142 itens, tendo 62 (81,5\% de 76) desses itens estado presentes em pelo menos um processo de aquisição.

Por fim, cabe ressaltar que do universo de itens analisados ( $n=142), 76(53,5 \%)$ estavam presentes nos processos de aquisição de alimentos e nos cardápios da alimentação escolar. É importante notar que os percentuais somados dos Grupos A, B e C ultrapassam os 100\% devido à sobreposição entre eles, ou seja, à presença de itens que fazem parte de simultaneamente de mais de um grupo.
A média aritmética simples da variedade de alimentos e preparações culinárias declarados para a Região Sul foi de $\bar{x}=16$, sendo a mediana $M d=15$ e o desvio padrão $\mathrm{DP}=7,2$. A variedade mínima de alimentos e preparações culinárias regionais foi de 5 na Mesorregião Oeste Paranaense e a máxima de 31 na Mesorregião Centro Oriental Rio Grandense, obtendose a amplitude de 26. A média aritmética simples do estado do Paraná foi $\bar{x}=12,9$, a de Santa Catarina foi de $\bar{x}=17,8$ e a do Rio Grande do Sul foi de $\bar{x}=18$.

O Gráfico 1 mostra a variedade/diversidade de alimentos e preparações culinárias regionais presentes nos processos de aquisição dos 21 municípios da pesquisa.

Gráfico 1. Variedade de itens por grupo e variedade de itens presentes nos documentos (cardápios e processos de aquisição)

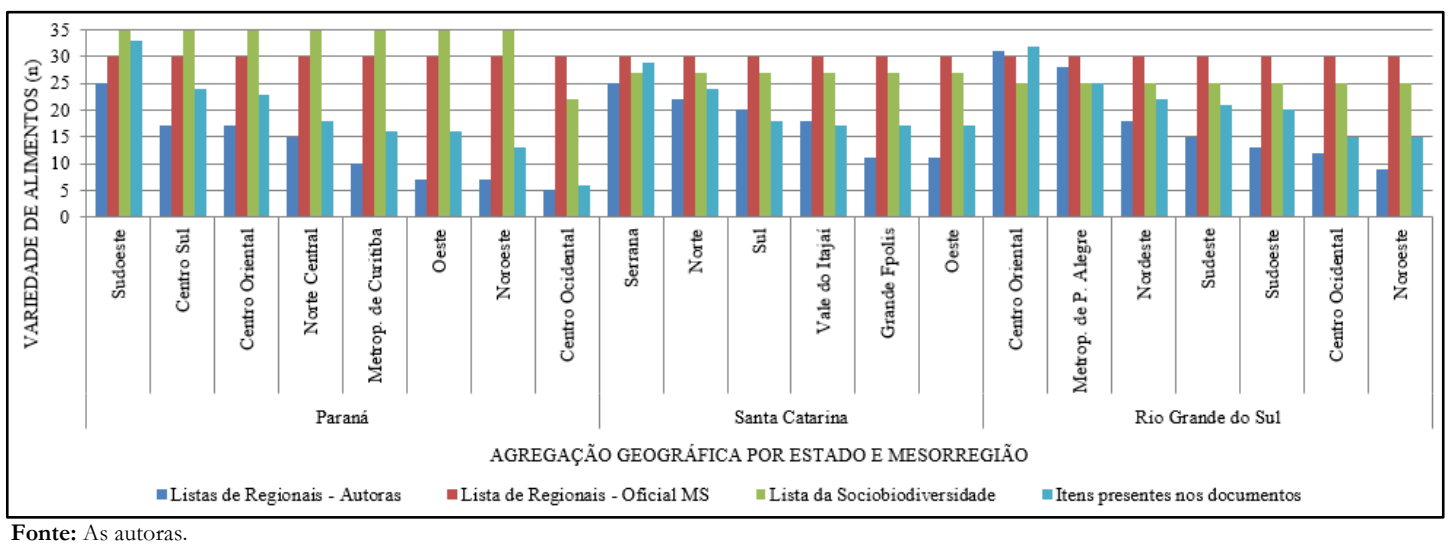

A média aritmética simples da variedade de itens presentes nos documentos dentre o universo dos 142 itens pesquisados (Grupo A, B e C) da Região Sul foi de $\bar{x}=20,04$, sendo a mediana $M d=18$ e o desvio padrão $\mathrm{DP}=5,9$.

A variedade mínima na aquisição de produtos foi de 6 na Mesorregião Centro Ocidental Paranaense e a máxima de 33 na Mesorregião Sudoeste Paranaense, obtendo-se a amplitude de 27. A média aritmética simples do estado do Paraná foi de $\bar{x}=18,6$, a de Santa Catarina foi de $\bar{x}=19,8$ e a do Rio Grande do Sul foi de $\bar{x}=21,4$.
Dos 35 alimentos da Lista da Sociobiodiversidade, apenas três foram identificados nos processos de aquisição dos municípios pesquisados, sendo eles o maracujá, a erva-mate e o pinhão. Desses, o maracujá foi o único alimento comum aos processos de aquisição dos três estados da Região Sul, o que representa $42 \%$ $(\mathrm{n}=9)$ municípios. 
Destaca-se o papel da agricultura familiar na oferta desse alimento, uma vez que em municípios de seis mesorregiões ${ }^{14}$ esse alimento constava nos editais de chamada pública $(66,6 \%)$.

A erva-mate, também um produto da sociobiodiversidade, foi adquirida por 47,6\% $(n=10)$ dos municípios pesquisados. Destes, sete se localizavam em mesorregiões do Paraná ${ }^{15}$ e três de Santa Catarina. ${ }^{16}$ Ressalta-se que, em todos os casos, a erva-mate foi identificada em processos de aquisição na modalidade licitação, em sua forma beneficiada e/ou industrializada. Desses três produtos, o pinhão foi o produto da sociobiodiversidade menos identificado nos municípios pesquisados, aparecendo em apenas um processo de aquisição de uma mesorregião de Santa Catarina, na modalidade chamada pública. ${ }^{17}$

Como é possível observar no Gráfico 2, dentre os 20 alimentos e preparações (produtos) mais presentes nos processos de aquisição dos municípios pesquisados, os cinco identificados em maior proporção por meio de chamada pública foram: mandioca, alface, batata-doce, couve-folha e repolho. Os alimentos lentilha, erva-mate e canela foram identificados somente em processos de aquisição na modalidade licitação.

Gráfico 2. Relação entre a lista dos 20 alimentos e preparações (produtos) mais presentes nos processos de aquisição dos municípios pesquisados e o percentual dos processos de aquisição por modalidade (licitação ou chamada pública)

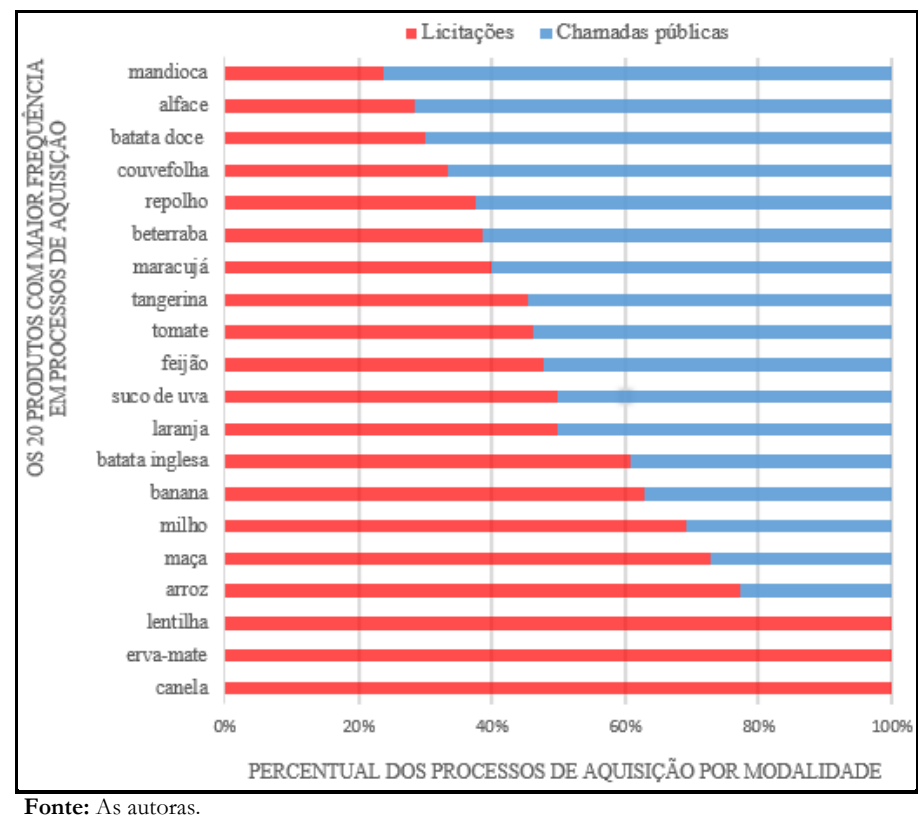

Do total de 142 produtos, 123 eram passíveis de serem localizados em processos de aquisição (dezenove dos 142 eram do tipo de preparações, que não poderiam ser localizados nos processos de aquisição; por exemplo, stollen e strudel). Desses 123 produtos, os 20 com a maior "variedade de

${ }_{14}$ Noroeste, Centro Oriental e Norte Central Paranaense, região Metropolitana de Curitiba (PR), Sul Catarinense e Grande Florianópolis (SC).

15 Noroeste, Sudoeste, Oeste, Centro Sul, Centro Ocidental e Centro Oriental Paranaense, região Metropolitana de Curitiba.

${ }^{16}$ Norte, Oeste e Serrana Catarinense. identificação" (ou presença) ${ }^{18}$ nos processos de aquisição dos municípios da amostra estão apresentados no Gráfico 3, classificados em ordem decrescente de identificação. A beterraba foi o alimento mais presente, aparecendo em 19 dos 21 municípios. A Análise de Pareto demonstra elevada concentração de

\footnotetext{
17 Serrana Catarinense.

18 "Variedade de identificação" diz respeito ao número de vezes que um determinado alimento foi identificado em um processo de aquisição. Por exemplo, suponhamos que o alimento mandioca tenha sido identificado em três municípios. Logo, sua variedade de identificação é igual a três.
} 
presença em reduzido número de produtos nos processos de aquisição. Os 12 produtos mais presentes equivalem a aproximadamente $60 \%$ da totalidade dos processos de compra (sendo que os demais 111 produtos equivalem a 40\%).
Do total de alimentos regionais e alimentos da sociobiodiversidade presentes nos processos de aquisição ( $\mathrm{n}=76$ ), identificou-se aqueles inseridos nas modalidades chamada pública e licitação. Do total desses alimentos, $47,7 \%$ estavam presentes nos processos de aquisição na modalidade chamada pública.

Gráfico 3. "Variedade de Identificação" dos produtos nos processos de aquisição dos municípios, Análise de Pareto

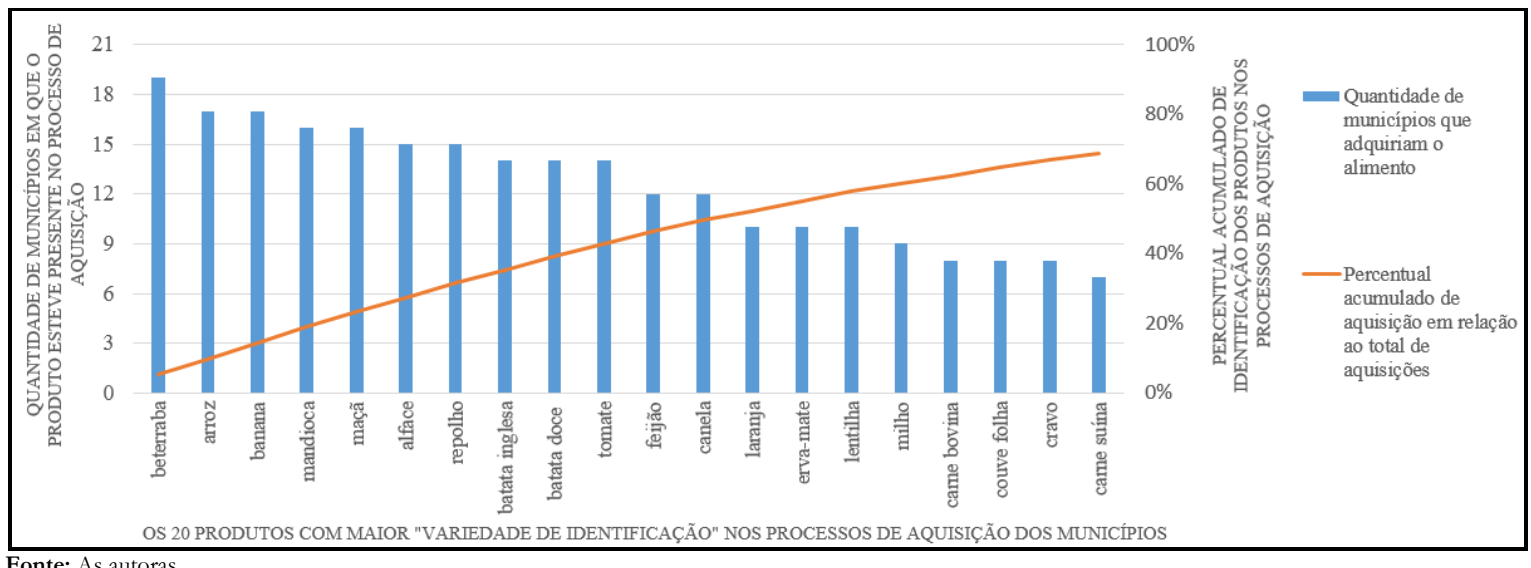

Em relação às 19 preparações culinárias regionais (somente preparações culinárias, não incluindo alimentos que também podem ser preparações) que constam no grupo de Listas de Regionais - Autoras, o município representante da mesorregião Vale do Itajaí (SC) foi o que declarou como regionais a maior quantidade de preparações $(\mathrm{n}=5)^{19}$, seguido pelos municípios representantes das mesorregiões Centro Oriental e Centro Sul Paranaense (PR) e Sudoeste, Noroeste e Centro Ocidental Rio Grandense (RS), cada um desses apresentando três declarações sobre preparações regionais. Churrasco foi a preparação culinária regional mais citada em todos os municípios pesquisados $(n=6)$, seguido pelo carreteiro $(\mathrm{n}=4)$. Ensopado de mandioca e chucrute foram citadas duas vezes e as demais preparações foram citadas apenas uma vez ${ }^{20}$. Do total de 19 preparações culinárias regionais declaradas pelos atores do PNAE, foram identificadas nos cardápios analisados apenas 8 preparações $(42,1 \%)^{21}$.

\section{DISCUSSÃO}

Este estudo analisou e identificou como os alimentos e preparações culinárias regionais e os alimentos da sociobiodiversidade estão presentes na alimentação escolar de municípios representantes de 21 mesorregiões da região Sul do Brasil. Na literatura científica foi encontrado, até o momento, apenas um estudo de caso que buscou identificar previamente os alimentos regionais para posterior análise da sua inserção na alimentação escolar, tendo em vista o caráter restritivo da lista pré-existente[32]. Não foram encontrados estudos que avaliassem a inserção de alimentos da sociobiodiversidade no PNAE, o que demonstra o caráter inédito e o potencial de contribuição desse tipo de pesquisa para a literatura científica.

${ }^{19}$ Chucrute, nhoque de batata, schwarsaur (sopa de sangue), stollen, strudel.

20 Arroz com galinha, canja/sopa de frango, ensopado de mandioca, feijoada, pirão de peixe, porco no tacho e virado de feijão.

21 Arroz com galinha, canja/sopa de frango, carreteiro, ensopado de mandioca, feijoada, pirão de peixe, porco no tacho, virado de feijão. 
Como primeiro achado, verifica-se a importância da etapa de entrevistas para a definição dos alimentos regionais em âmbito municipal. Isso se deve ao fato de que os municípios da Região Sul, apesar de possuírem aspectos em comum, apresentam especificidades em termos de colonização, características territoriais, clima, vocação agrícola, dentre outros.

Uma vez que o Programa possui como uma de suas diretrizes respeitar e valorizar a cultura alimentar local[25], bem como dinamizar a economia local por meio da compra de alimentos produzidos pelo setor agrícola familiar [30], verifica-se a necessidade da elaboração de listas de alimentos e preparações regionais específicas aos variados contextos locais nos quais PNAE é executado.

A lista elaborada pelas autoras a partir das entrevistas com atores do PNAE nos 21 municípios pesquisados apresentou número consideravelmente superior $(\mathrm{n}=95)$ àquele disponibilizado na lista de alimentos regionais publicada pelo Ministério da Saúde $(n=30)^{[33]}$. Houve uma coincidência de 14 itens entre essas listas, ou seja, pode-se afirmar que 46,6\% dos produtos presentes na lista do MS estavam também presentes na lista elaborada pelas autoras (subgrupo B $\cap \mathrm{C})$.

Ressalta-se a abrangência desta pesquisa em termos de território e de representatividade geográfica. Este estudo abarcou 21 das 23 mesorregiões da Região Sul do Brasil.

Um dos elementos originais da pesquisa deveu-se à utilização de três grupos de listas para proceder a identificação dos alimentos e preparações nos processos de aquisição e nos cardápios da alimentação escolar, sendo uma delas elaborada a partir das entrevistas com os atores do PNAE nos municípios e as outras duas extraídas de listas oficiais pré-existentes de alimentos regionais e da sociobiodiversidade.

A análise dos processos de aquisição visando a identificação dos produtos da sociobiodiversidade demonstrou que sua incorporação na alimentação escolar é demasiadamente reduzida, uma vez que dos 35 alimentos listados para essa região, apenas $3(8,5 \%)$ estiveram presentes nos documentos analisados. Além disso, os produtos da sociobiodiversidade tiveram baixa representatividade no total de processos de aquisição, quando comparados ao percentual relativo de seus itens. Soma-se a isso o fato de que o produto mais presente nos processos de aquisição dos municípios pesquisados, a erva-mate, foi identificada somente na modalidade licitação.

Considerando que a modalidade chamada pública é o procedimento administrativo voltado à seleção de proposta específica para aquisição de gêneros alimentícios provenientes da agricultura familiar ${ }^{[39]}$, esse dado demonstra que os produtores desse setor provavelmente não estiveram presentes na venda do produto para a alimentação escolar no período pesquisado, descaracterizando, nesse caso, a erva-mate como produto da sociobiodiversidade.

Uma vez que dos 35 alimentos presentes na Lista da Sociobiodiversidade, somente o pinhão e o maracujá foram citados pela população entrevistada como alimentos regionais, identificou-se a necessidade de se fomentar a produção dos alimentos da biodiversidade brasileira por agricultores familiares e integrantes de comunidades tradicionais e o seu consumo pela população. Entretanto, deve-se considerar que o caráter recente da Portaria Interministerial no $163 / 2016{ }^{[38]}$ pode ser um dos fatores que contribuíram para a baixa presença desses alimentos nas entrevistas e nos processos de aquisição e cardápios da alimentação escolar.

Por meio das análises, foi possível perceber que os dez alimentos mais presentes nos processos de aquisição ${ }^{22}$ encontravam-se em processos de aquisição na modalidade chamada pública, indicando a participação da agrícola familiar na comercialização desses produtos.

No entanto, foi possível identificar que em 66,6\% dos casos, a inclusão dos alimentos da sociobiodiversidade na alimentação escolar não se deu da forma preconizada pela legislação ${ }^{[39]}$, pois esses alimentos estiveram presentes em processos de aquisição na modalidade licitação, sendo provável baixa

22 Mandioca, alface, batata-doce, couve-folha, repolho, beterraba, maracujá, tangerina, tomate e feijão. 
ou nula a participação da agricultura familiar por essa modalidade.

Ressalta-se que a inclusão dos produtos da sociobiodiversidade nos cardápios da alimentação escolar tem o potencial de incentivar a formação de cadeias produtivas de interesse de povos tradicionais e agricultores familiares, valorizando as suas práticas e saberes e assegurando os seus direitos ${ }^{[40]}$.

Essa iniciativa é considerada um grande avanço em termos de políticas públicas ao incentivar os agricultores familiares e de comunidades tradicionais a integrar o mercado institucional de alimentos ${ }^{[41]}$.

Destaca-se que o Brasil foi considerado pela ONU um exemplo a ser seguido[22] pela inovação na criação de programas como o Plano Nacional para a Promosão das Cadeias de Produtos da Sociobiodiversidade, a partir do qual procura-se introduzir esses alimentos na alimentação escolar.

A análise dos itens considerados preparações culinárias regionais encontradas apenas nos cardápios ${ }^{23}$ declaradas nas entrevistas demonstrou a reduzida quantidade de preparações regionais nos cardápios da alimentação escolar. A maioria das preparações esteve presente nenhuma $(n=0)$ ou uma $(n=1)$ vez nos cardápios analisados.

Por outro lado, quando analisados os alimentos/preparações culinárias regionais, que poderiam ser encontrados tanto nos processos de aquisição quanto nos cardápios, identificou-se o arroz e o feijão entre os três alimentos/preparações com maior presença nos processos de aquisição dos três estados pesquisados.

A análise das preparações regionais nos cardápios demonstrou dificuldade desse tipo de avaliação, considerando os diferentes padrões de cardápio, e principalmente o reduzido grau de especificação e detalhamento dos mesmos. De maneira que muitos alimentos regionais podem não ter sido identificados, uma vez que as preparações e ingredientes das mesmas não estavam completos (por exemplo, os termos salada, fruta e legume sem a identificação dos mesmos). Isso poderia explicar o

23 Arroz com galinha, batata doce caramelizada, broa de milho, canja/sopa de frango, capeletti, carreteiro, chucrute, churrasco, ensopado de mandioca, feijoada, número inferior de alimentos e preparações regionais nos cardápios quando comparado com o percentual desses mesmos itens no processo de aquisição.

Apesar do cuidado de se apresentar a definição de alimentos regionais adotado nessa pesquisa aos entrevistados no momento da coleta, eventualmente os mesmos parecem ter referido como regional somente aquele alimento produzido no local, sem considerar o contexto histórico e cultural do alimento.

Desse modo, em alguns municípios, a soja foi citada como regional por ser produzida no local, apesar de não fazer parte do hábito de consumo da população. O seu consumo não faz parte da cultura brasileira, sendo considerado incipiente. Assim, a preferência pela soja não tem representação em nenhuma região específica do país ${ }^{[42]}$. Ainda assim, foi mencionada como alimento regional em cerca de 19\% dos municípios pesquisados, possivelmente por associação à produção local do grão (Paraná e Rio Grande do Sul estão entre os maiores produtores em nível nacional) ${ }^{[43]}$.

Por outro lado, os entrevistados podem não ter lembrado de mencionar todas as possibilidades de alimentos regionais, a exemplo da erva-mate, que não foi citada em nenhum município. Esta é uma planta nativa da Região Sul do continente americano e seu uso remonta a centenas de anos. Por suas características naturais de distribuição geográfica ${ }^{[44]}$ e por sua grande importância econômica, ambiental e cultural[ ${ }^{[4]}$, até hoje representa um elo entre os estados do Sul do Brasil e é reconhecidamente um alimento regional nesses estados ${ }^{[4]}$, onde a maior parte é produzida por agricultores familiares ${ }^{[45]}$.

Sabe-se que a técnica utilizada para a elaboração das listas de alimentos regionais a partir das entrevistas depende da memória dos indivíduos. No entanto, visando superar ao máximo essa limitação, a presente pesquisa buscou trabalhar com lista de alimentos regionais já existente e, junto a isso, identificar os alimentos regionais com atores-chave de diferentes setores do PNAE.

Esses achados apontam para a necessidade de aplicação de instrumentos mais precisos para a coleta dos dados. $\mathrm{O}$ instrumento desenvolvido recentemente 
por Fabri e colaboradoras ${ }^{[32]}$ para a identificação de alimentos regionais apresenta-se como uma sugestão de aprimoramento da metodologia que pode ser utilizada para a condução de futuros estudos.

Outra possível limitação do presente estudo foi a impossibilidade de se obter nos processos de aquisição analisados os nomes científicos dos alimentos regionais e da sociobiodiversidade presentes na Lista de Regionais - Oficial MS e na Lista da Sociobiodiversidade, o que pode ter diminuído a acurácia das informações obtidas junto aos documentos.

Ressalta-se a importância de se aprofundar as pesquisas relacionadas aos alimentos regionais para que se possa ampliar a diversificação de itens na alimentação escolar. De forma complementar, aponta-se para a necessidade de maior conscientização acerca da importância da introdução de alimentos regionais na alimentação escolar como forma de promoção da identidade cultural, aceitabilidade das preparações e facilidade de acesso aos locais ${ }^{[31,32]}$.

Considerando-se que é diretriz da alimentação escolar o uso de alimentos variados e que respeitem a cultura $\mathrm{e}$ as tradições ${ }^{[25]}$ sugere-se que sejam consideradas iniciativas governamentais visando a ampliação da diversidade dos alimentos adquiridos para o PNAE na Região Sul.

Os resultados da pesquisa dão indícios da necessidade de intensificação de iniciativas no sentido de ampliar a conscientização e capacitação acerca da importância da aquisição de alimentos regionais provenientes da agricultura familiar e de alimentos da sociobiodiversidade. Apesar de já existirem incentivos financeiros para a priorização dos produtos da agricultura familiar, sugere-se que incentivos complementares visando o mesmo objetivo sejam incorporados ao PNAE.

Sugere-se que haja a coordenação de esforços entre o Fundo Nacional de Desenvolvimento da Educação (FNDE/MEC) e as entidades executoras do PNAE - estados ou municípios - para a condução de levantamentos acerca dos alimentos e preparações regionais e dos produtos da sociobiodiversidade locais pelos nutricionistas (responsáveis técnicos) para que tais itens sejam inclúdos com maior frequência nos cardápios.
Além disso, sugere-se que esses mesmos agentes -FNDE/MEC e entidades executoras - dentro de suas respectivas competências - aprimorem as atividades de monitoramento da diversidade de alimentos adquiridos, incorporando aspectos relacionados à quantidade e periodicidade, além de especificar a modalidade de aquisição e os preços praticados.

Devido à extensão do programa e ao alcance do PNAE, sugere-se que esse monitoramento seja feito por meio de sistemas automatizados centralizados e estruturado com base em indicadores oficiais de qualidade.

\section{REFERÊNCIAS}

[1] WHO/UNCCD. Our Planet, Our Health, Our Future. Human health and the Rio Conventions: biological diversity, climate change and desertification. Genebra: WHO \& UNCCD; 2012.

[2] Nações Unidas. ACC/SCN. Fourth Report on the World Nutrition Situation. Genebra: ACC/SCN in collaboration with IFPRI; 2000.

[3] Rittenschober D, Charrondiere RU. Report on the Nutrition Indicators for Biodiversity - Food Composition and Food Consumption: Global Progress Report. Roma: FAO/INFOODS; 2013.

[4] WHO. Secretariat of the Convention on Biological Diversity. Connecting Global Priorities: Biodiversity and Human Health: A State of Knowledge Review. Genebra: WHO; 2015.

[5] UNEP. The Critical role of global food consumption patterns in achieving sustainable food systems and food for all. Paris: UNEP; 2012.

[6] Burlingame B, Dernini S. Sustainable Diets and Biodiversity: Directions and Solutions for Policy, Research and Action. Roma: FAO; 2010.

[7] Chappell MJ, Lavalle L. Food security and biodiversity: Can we have both? An agroecological analysis. Agriculture and Human Values. 2011;28(1):3-26.

[8] Coradin L, Siminski A, Reis A. Espécies nativas da flora brasileira de valor econômico atual ou potencial: plantas para o futuro - Região Sul. Brasília: MMA; 2011.

[9] Burity V, Franceschini T, Valente F, Recine E, Leão M, et al. Direito humano à alimentação adequada no contexto da segurança alimentar e nutricional. Brasília(DF): ABRANDH; 2010. 
[10] Brasil. Conselho Nacional de Segurança Alimentar e Nutricional (CONSEA). A Segurança Alimentar e Nutricional e o Direito Humano à Alimentação Adequada no Brasil: indicadores e monitoramento da Constituição de 1988 aos dias atuais. Brasília: Consea; 2010.

[11] Santos F, Tonezer C, Rambo AG. Agroecologia e agricultura familiar: um caminho para a soberania alimentar? In: Anais do 47o Congresso da Sociedade Brasileira de Economia, Administração e Sociologia Rural [internet]. [acesso em $24 \mathrm{fev}$ 2017]. Porto Alegre: SOBER; 2009. Disponível em: http://www2.ufersa.edu.br

[12] Perrings C, Jackson L, Bawa K, Brussard L, Brush S, et al. Biodiversity in agricultural landscapes: saving natural capital without losing interest. Conservation Biology. 2010;20:263-264.

[13] Lairon D. Biodiversity and sustainable nutrition with a food-based approach. In: Proceedings of the International Scientific Symposium "Biodiversity and Sustainable Diets United Against Hunger"; 3-5 nov 2010; Rome. Rome: FAO; 2010.

[14] Batista Filho M, Batista LV. Transição alimentar e nutricional ou mutação antropológica? Revista Ciência Cultura. 2010;62(4):26-30.

[15] Triches RM, Schneider S. Alimentação escolar e agricultura familiar: reconectando o consumo à produção. Saúde Soc. 2010;19(4):933-945.

[16] FAO/OPS. América Latina y el Caribe: panorama de la seguridad alimentaria y nutricional. Santiago: FAO; 2017.

[17] Assis RL. Desenvolvimento rural sustentável no Brasil: perspectivas a partir da integração de ações públicas e privadas com base na agroecologia. Economia Aplicada. 2006;10:75-89.

[18] Brasil. Decreto no 7.272, de 25 de agosto de 2010. Regulamenta a Lei no 11.346 , de 15 de setembro de 2006, que cria o Sistema Nacional de Segurança Alimentar e Nutricional SISAN com vistas a assegurar o direito humano à alimentação adequada, institui a Política Nacional de Segurança Alimentar e Nutricional - PNSAN, estabelece os parâmetros para a elaboração do Plano Nacional de Segurança Alimentar e Nutricional, e dá outras providências. Brasília: Diário Oficial da União. 25 ago 2010.

[19] Schmitt CJ. Encurtando o caminho entre a produção e o consumo de alimentos. Revista Agriculturas: Experiências em Agricultura. 2011;8(3):4-8.

[20] Burlandy L, Gomes FS, Cravalho CMP, Dias PD, Henriques P. Intersetorialidade e potenciais conflitos de interesse entre governos e setor privado comercial no âmbito das ações de alimentação e nutrição para o enfrentamento de doenças crônicas não transmissíveis. Vig. Sanit. Debate. 2014;2(4):124-129.
[21] Nações Unidas/UNSCN Country study for the second International Conference on Nutrition (ICN2). Nutrition Impact of Agriculture and Food Systems: Brazil: UNSCN; 2013.

[22] Thompson B, Amoroso L. Improving Diets and Nutrition: Food-based Approaches. Roma: FAO; 2010.

[23] Santarelli M. Cooperação Sul-Sul brasileira: a experiência do Programa Nacional de Alimentação Escolar em Moçambique. Rio de Janeiro: ActionAid Brasil; 2015.

[24] Soares P, Martinelli SS, Melgarejo L, Cavalli SB. Fornecimento de alimentos da agricultura familiar para a alimentação escolar: o exemplo do Programa de Aquisição de Alimentos. Segur. Aliment. Nutr. 2013;20(1):41-51.

[25] Brasil. Lei no 11.947, de 16 de junho de 2009. Dispõe sobre o atendimento da alimentação escolar e do Programa Dinheiro Direto na Escola. Brasília: Diário Oficial da União. 16 jun 2009.

[26] Triches RM, Baccarin JG. Interações entre alimentação escolar e agricultura familiar para o desenvolvimento local. In: Teo CRPA, Triches RM. Alimentação escolar: construindo interfaces entre saúde, educação e desenvolvimento. Chapecó: Argos; 2016. p. 89-110.

[27] Brasil. Conselho Nacional de Segurança Alimentar e Nutricional - CONSEA. Princípios e Diretrizes de uma Política de Segurança Alimentar e Nutricional. Textos de Referência da II Conferência Nacional de Segurança Alimentar e Nutricional. Brasília: Consea, 2004.

[28] Sousa AA, Silva APF, Azevedo E, Ramos MO. Cardápios e sustentabilidade: ensaio sobre as diretrizes do Programa Nacional de Alimentação Escolar. Rev. Nutr. 2015;28(2):217229.

[29] Altieri MA, Nicholls CI. Agroecology scaling up for food sovereignty and resiliency. Sustainable Agriculture Reviews. 2012;11:1-29.

[30] Chaves LG, Mendes PNR, Brito RR, Botelho RBA. O Programa Nacional de Alimentação Escolar como promotor de hábitos alimentares regionais. Rev. Nutr. 2009;22(6):857-866.

[31] Gabriel CG, Costa LCF, Calvo MCM, Vasconcelos FAG. Planejamento de cardápios para escolas públicas municipais: reflexão e ilustração desse processo em duas capitais brasileiras. Rev. Nutr. 2012;25(3):363-372.

[32] Fabri RK, Proença RPC, Martinelli SS, Cavalli SB. Regional foods in Brazilian school meals. BFJ. 2015;117(6):1706-1719.

[33] Brasil. Ministério da Saúde. Secretaria de Atenção à Saúde. Departamento de Atenção Básica. Alimentos regionais brasileiros. Brasília: Ministério da Saúde; 2015. 
[34] Brasil. Instituto Brasileiro de Geografia e Estatística. Mesorregiões Brasil [internet]. [acesso em 03 mar 2017]. Disponível em: https://www.ibge.gov.br

[35] Cavalli S, Soares P, Martinelli SS, Fabri RK, Ebone MV, et al. Estratégias de Gestão da Qualidade dos Vegetais e Frutas Fornecidos pelo Programa de Aquisição de Alimentos (PAA) para a Alimentação Escolar. Relatório Final (Edital MCT/MDS - SAGI/CNPq no 36/2010, processo: 63662/2010-7). Brasília(DF): MCT/MDS; 2012.

[36] Handcock MS, Gile KJ. Comment: on the concept of snowball sampling. Sociological Methodology. 2011;41:367-371.

[37] Sichieri R. Dietary patterns and their associations with obesity in the Brazilian city of Rio de Janeiro. Obesity Research. 2002;10:42-49.

[38] Brasil. Ministério do Meio Ambiente. Portaria Interministerial no 163, de 11 de maio de 2016. Diário Oficial da União. 11 maio 2016.

[39] Brasil. Fundo Nacional de Desenvolvimento da Educação. Aquisição de produtos da agricultura familiar para a alimentação escolar. 2 ed. - versão atualizada com a Resolução CD/FNDE no 04/2015. Brasília: FNDE; 2016.

[40] Gomes RM, Lopes SRS. Aquisição de Produtos da Agricultura Familiar para a alimentação escolar. 2 ed. - versão atualizada com a Resolução CD/FNDE no 04/2015. Brasília: FNDE; 2016.

[41] Brasil. Ministério do Meio Ambiente. Iniciativa estimula economia extrativista [internet]. 2016 [acesso em 11 jan 2017]. Disponível em: http:/ /www.mma.gov.br

[42] Neves CM. Possibilidades e dificuldades do uso da soja in natura na alimentação humana no Brasil. Porto Alegre: UFRGS; 2003.

[43] Barbosa MZ, Assumpção R. Ocupação territorial da produção e da agroindústria da soja no Brasil nas décadas de 80 e 90. Informações Econômicas. 2001;31(11):7-17.

[44] Boguszewski JH. Uma história cultural da erva-mate: o alimento e suas representações. Paraná: UFPR; 2007.

[45] Pasinato R. Aspectos etnoentomológicos, socioeconômicos e ecológicos relacionados à cultura da erva-mate (Ilex paraguariensis) no município de Salto do Lontra, Paraná, Brasil. [dissertação]. Piracicaba: Universidade de São Paulo; 2003. 